\title{
Airborne System for Pipeline Surveillance Using an Unmanned Aerial Vehicle
}

\author{
Mbadiwe Benyeogor, Adeboye Olatunbosun, and Sushant Kumar
}

\begin{abstract}
Our work involves the development of a quadcopter Unmanned Aerial Vehicle (UAV) system with remote sensors onboard for monitoring oil and gas pipelines. Two Liquefied Petroleum Gas (LPG) sensors were used for LPG gas leakage detection. The Multiwii software is used to control, track and simulate the 3D motion of the UAV in flight. Using this device, experimental data from field tests were analyzed with MATLAB. Results reveal that the developed system has performed as expected. Thus, our device can be used to enhance asset monitoring and operational safety in the oil industry.
\end{abstract}

Index Terms-Aerial Vehicle, Flight Control, Flammable Gases, Remote Sensing, Sensors Remote.

\section{INTRODUCTION}

Occasional leakage of oil and gases due to equipment failure, during exploration and production processes, is commonplace in the oil and gas industry. The frequent occurrence of these incidents is capable of polluting the environment, endangering lives and disrupting the agricultural activities of the local community. Also, the inhalation of the gases emanating from the leakages is deleterious to health. Traditional methods of inspection involve the use of handheld devices to inspect pipelines for leakages; however, these usually expose people to a hazardous working condition that could lead to an accident. A notable instance is the 18th October 1998 petroleum pipeline explosion at Jesse in Niger Delta, Nigeria which was said to be the worst of several similar incidents in Nigeria [1]

To address these problems, we propose an aerial remote sensing technique for detecting Volatile Organic Compound (VOC) emissions from pipeline leaks; this incorporates two Liquefied Petroleum Gas (LPG) Gas Sensors System that is embedded on an unmanned aerial vehicle (UAV). The UAV could be remotely controlled and monitored from a ground station while flying overhead along the oil and gas pipeline routes with the capability of detecting and reporting any oil or gas leakage along the routes. The LPG sensors measure the concentration of VOC leaks in the atmosphere near the oil and gas pipeline; the data from these sensors are

Published on February 14, 2020.

M. Benyeogor is with the Faculty of Physics and Electrical Engineering University of Bremen, NW1 Otto-Hahn-Allee 1, 28359 Bremen, Germany (e-mail: samrexbenzil@gmail.com).

A. Olatunbosun is with the Department of Electrical and Electronic Engineering, Faculty of Technology, University of Ibadan, Ibadan, Nigeria (e-mail: a.olatunbosun@ui.edu.ng).

S. Kumar is with the Faculty of Physics and Electrical Engineering University of Bremen, NW1 Otto-Hahn-Allee 1, 28359 Bremen, Germany (e-mail: sushant@uni-bremen.de). processed by a dedicated in-flight signal processor which has been programmed to generate the information about the state of the environment being monitored and relay it to the ground station via radio telemetry.

\section{LITERATURE REVIEW}

Several surveillance operations require the application of UAV systems; oil and gas pipeline monitoring are a quintessential example of this application [2]. According to Austin, the UAV commonly known as a drone is simply a flying machine without a human pilot on board [3]. In remote sensing, the UAV could serve as an efficient airborne instrument deployment system. The quadcopter is a type of UAV in the form of a helicopter with four rotors. It has generated several research areas of interest in recent times and has found several applications especially in the area of environmental monitoring. Its application as a monitoring system has become very relevant in the oil and gas industry where safety measures are sacrosanct. According to Gómez and Green [4], what the oil and gas industry is seeking is to be able to use small unmanned aircraft to patrol oilfields. Currently, the oil and gas industry trends toward the usage of UAVs for onshore pipeline and facility surveillance. Current research works in this area focus on how to optimally improve and miniaturize existing surveillance systems and then mount them on a UAV for effective patrol of infrastructures. To this effect, new avionics, measurement instruments, and control techniques have been developed [5].

Before now, several researchers have done substantial works in the development of mobile remote sensing systems for monitoring oil and gas infrastructures. For example, Ejofodomi explored the feasibility of using small mobile robots for early detection of ground oil leakage, a methodology defined as Ground Robotic Oil Spill Surveillance (GROSS) [6]. Also, Ramon et al. [7], developed a mobile platform called 'Turtlebot' for detecting gas leakages. The control of the mobile base and the inspection device was integrated with a Robot Operating System (ROS).

Oil and gas leak sensing Mobile Ground Robots (MGR) is effective within well-structured oil and gas refinery and storage facility environments but is physically challenged when it comes to gaining access to pipeline routes that run through swamps and thick bushes. Being that a quadcopter is a flying machine that can be boarded with VOC detectors to fly over impassable terrains to the relevant locations to be scanned, many developers of mobile VOC detection systems are now using quadcopter UAV instead of MGR [5]. This concept was emphasized by Kelvin [8], who explained why 
advances in mobile remote sensing and UAV technology are well situated for monitoring the Trans-Alaskan Pipeline. The aerodynamics of the quadcopter makes it the choicest UAV because it flies with a lower speed and can be controlled to hover a particular location on the ground compared to a fixed-wing UAV which has a higher speed and cannot be suspended in the mid-air. Based on this concept, Omijeh et al. [9], developed a quadcopter system with a payload for pipeline inspection.

Based on the fact that most petroleum pipelines run through creaks and unmotorable topographies, moderate speed and maneuverable UAV like the quadcopter is required for their inspection. In our research, a similar device like the VOC sensing device employed by Ejofodomi and Ofualagba in [6] is developed. Like that of Omijeh et al. in [9], this was embedded on the quadcopter UAV to form an airborne instrumentation system that could enhance the surveillance of inaccessible pipeline routes. Unlike many similar works, our work entails methodical ways of using the UAV for pipeline monitoring in real-time. To enhance our airborne objectives, we have deemed it necessary to adopt only Micro/CMOS (Complementary Metal Oxide Semiconductor) based sensors and avionic devices, bearing in mind the size and weight factor. Details of this are expounded in the remaining parts of this paper.

\section{METHODOLOGY}

\section{A. The Aerial Vehicle}

Our gas leak detection system becomes relevant once the UAV can go airborne and fly overhead the pipeline route to be surveyed. Thus, half of our work is devoted to developing an airworthy UAV that can reliably deploy instruments to any given location to be surveyed. The design of a quadcopter was adopted for our UAV based on the work of Kotarski et al. [10] and Modi [11]. Its overall flight mechanism and control system is modeled in Fig. 1. This UAV comprises four motors. Each of these motors is controlled by the flight controller through a special servomechanism called the Electronic Speed Controller (ESC) - motor driver which working is based on the principle of Pulsed Width Modulation (PWM).

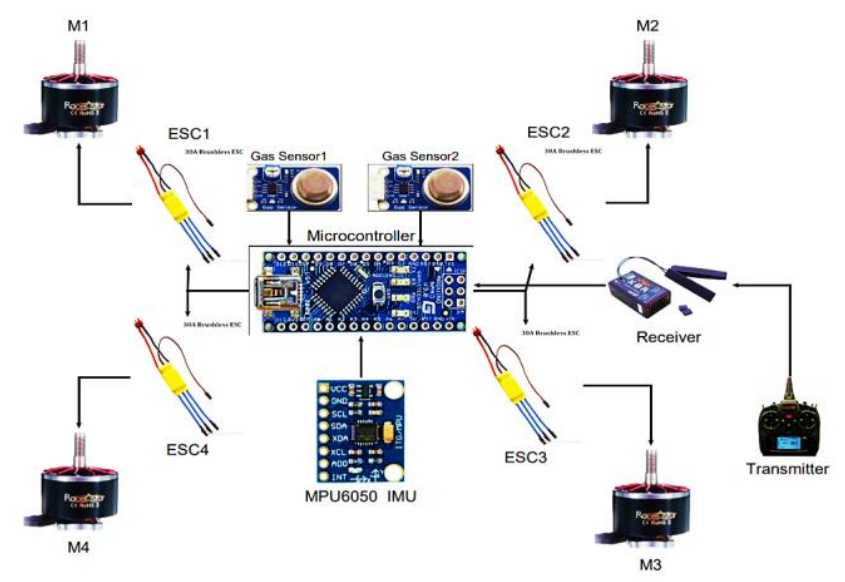

Fig. 1 Model of the aerial vehicle system

\section{B. Propulsion Power and Thrust}

For the design of our UAV propulsion system, the estimation of static thrust was carried out. This is to ensure that the right propellers, motors, and the battery is selected, for our UAV to be capable of self-lift-off. Firstly, the size of power that is transmitted by the motors to the propellers is calculated in terms of Revolution-Per-Minute (RPM) using eq.1.

$$
\text { Power, } P=K_{p} \cdot R P M^{\text {Power factor }}
$$

Where the term $K_{p}$ is the propeller constant. $K_{p}=0.090$ while Power factor $=3.2$.

$$
\begin{aligned}
& \text { Motor } R P M=K V \times \text { Battery } E M F \\
& \quad=1000 \frac{r p m}{v} \times 11.1 v=11,100 \mathrm{rpm} \\
& \therefore P=0.090 \times 11.1^{3.2} \approx 199.2 \mathrm{watt}
\end{aligned}
$$

In principle, the thrust produced by the propeller is given by:

$$
T=\frac{\pi}{4} D^{2} \rho v \Delta v=\frac{\pi}{4} D^{2} \rho(\Delta v)^{2}
$$

If $\Delta v=\frac{2 P}{T}$, the eq. 4 becomes:

$$
T=\frac{\pi}{8} D^{2} \rho\left(\frac{2 P}{T}\right)^{2}
$$

By solving eq.5, we derive eq.6.

$$
T=\sqrt[3]{\frac{\pi D^{2} \rho P^{2}}{2}}
$$

According to our design;

$D=9^{\prime \prime}, \rho=1.225 \frac{\mathrm{kg}}{\mathrm{m}^{3}}, P=199.2$ watt.

Thus, the value of the thrust, $T$ generated by each motorpropeller system is evaluated as:

$T=183.56 N$

For four motors the resultant thrust $T_{R}$ is then:

$T_{R}=4 \cdot T=734.24 N$

To calculate the maximum mass, $m$ of the UAV system our propulsion engine can lift-off, we used Newton's equation in eq. 7 .

$$
m=\frac{T_{R}}{g}
$$

Hence, $m \approx \frac{734.24}{9.8}=74.922 \mathrm{~kg}$

The value of $m$ is used to determine the total mass of our UAV system, with payload inclusive, that can be sent airborne based on our design parameters.

\section{Avionics and Flight Control}

Based on the control concept Kotarski et al. [10], the flight controller is developed as a functional avionic system using the Atmel ATMEGA328 microcontroller (i.e. Arduino Nano), an MPU-6050 IMU, an HC-05 Bluetooth module, four ESC and a radio transmitter/receiver pair. The IMU 
integrates a triaxial gyroscope and an accelerometer on the same chip. These measures the attitudes and the angular accelerations of the quadcopter in-flight. The circuit diagram of the flight controller is shown in Fig. 2(a). The controller provides for four control Channels: throttle, roll, pitch, and yaw. The open-source Multiwii flight control software was used to develop the source files which coordinate the flow of signals for control and communications in the UAV flight system. By activating relevant library functions and adjusting the values of the PID control parameters in the flight control source file of the Multiwii software, the flight controller algorithm was implemented. This software along with the supporting library functions was uploaded to the flight controller. The physical construction of the flight controller is shown in Fig. 2 (b).

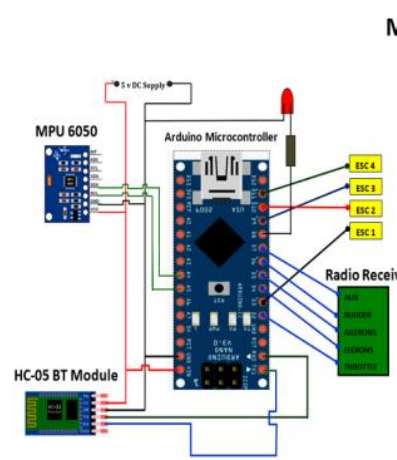

a circuit board

\section{Leak Detection Unit}

The Leak Detection System (LDU) is the instrumentation payload onboard the UAV, which measures the concentration of VOC near the UAV and telemeters this information to the ground station. This comprises the aforementioned two Liquefied Petroleum Gas (LPG) sensors. The LPG sensor is suitable for measuring the aerial concentration of smoke, ethanol, propane, butane, carbonmonoxide, kerosene- and gasoline- vapor. The MQ2 brand of LPG sensors is the most common. They can detect gas concentrations anywhere from 200 to $10000 \mathrm{ppm}$. At the core of every LPG-sensor, is a heating coil in a partially enclosed space. This enhances small stoichiometric combustions of the targeted gaseous hydrocarbons and oxygen, within the enclosure. The average temperature of this space (as measured by an on-chip resistance thermometer) varies linearly with the concentration of the gas. Using a Wheatstone bridge, this temperature is converted into a voltage variable, from which the value of gas concentration be computed [12]. We adopted the CMOS-based MQ2 LPG sensor for our work. Our LDU incorporates two PLG sensors and it is built around a dedicated signal processor, which is integrated into the flight control system. This signal processor is programmed to fuse data from these two gas sensors into a piece of comprehensive information about the chemical profile of the location being surveyed. The resulting information is radioed to the ground station by Bluetooth telemetry through the flight controller. The LDU responds reliably to any leakage of VOC in the vicinity of the UAV system and signals danger to the ground station whenever it measures an LPG concentration of 400 ppm or higher.

\section{E. Experimental Setup}

To ascertain the real-life applicability of our device, several tests were performed in a partially controlled environment. A major apparatus for this test is the complete aerial vehicle system, which is assembled from prefabricated parts as shown in Fig. 3, with the leak detection system as the payload. The mass of the UAV is 15.23 Kg while its total mass (with that of the LDU payload included) is $22.36 \mathrm{Kg}$. To determine the airworthiness of this UAV system, it was extensively tested in different meteorological conditions and environments, while its flight dynamics is remotely visualized with the Multiwii flight control interface.

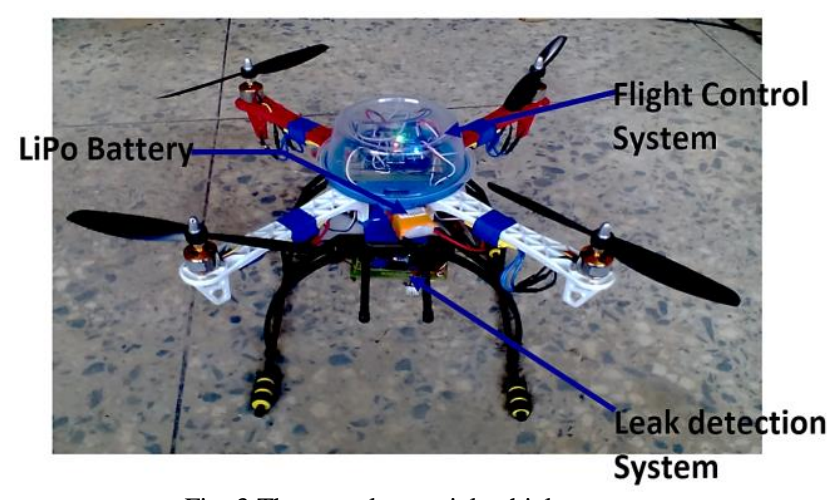

Fig. 3 The complete aerial vehicle system

Two experiments were conducted in the field to test the performance of the UAV with the gas detection system onboard. The first experiment was conducted to determine the linearity of the LDU, while the second experiment was carried out to determine the sensitivity of the LDU to VOC leakages while traversing along pipelines. For the first experiment (i.e. experiment-1), the setup comprises an LPG cylinder, piped to a valve-controlled nozzle connected to a gas manometer, as described in Fig. 4.

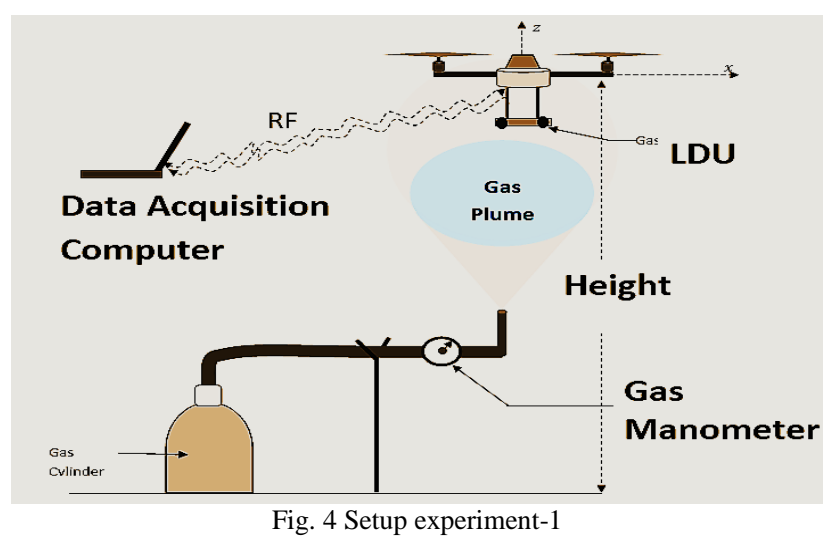

The manometer measures the initial pressure, $P_{g}$ of the LPG in the cylinder; while the LDU onboard the hovering UAV measures the concentration, $C_{g}$ of the LPG or any VOC at the instant of expulsion from the cylinder. Initially, a domestic LPG cylinder is filled with natural gas up to a maximum pressure of $2645 \mathrm{Nm}^{-2}$, after which it is allowed to leak-off its gaseous content and drop continually to 
successive lower values of pressure. The concentration of natural gas leaks in the air is measured by the LDU onboard the UAV at a constant height of 5 meters above the ground. Data about the concentration of expelled gases is processed by the LDU and transmitted to the remote computer in the ground station via Bluetooth telemetry. The measurements of experiment-1 are presented in Table I.

TABLE I: EXPULSION FLOW-RATE AGAINST MEASURED CONCENTRATION IN THE AIR AT HEIGHT OF 5 METERS ABOVE THE GROUND

\begin{tabular}{ccccc}
\hline \hline S/N & $\begin{array}{c}\text { Gas Flow } \\
\text { Rate }\left(\mathrm{Nm}^{-2}\right)\end{array}$ & $\begin{array}{c}\text { Gas Conc. In } \\
\text { Air }(\mathrm{ppm})\end{array}$ & $\begin{array}{c}\text { Gas Conc. In } \\
\text { Air }(\mathrm{ppm})\end{array}$ & $\begin{array}{c}\text { Gas Conc. } \\
\text { In Air } \\
(\mathrm{ppm})\end{array}$ \\
\hline 1 & 2645 & 210 & 223 & 216 \\
2 & 2552 & 250 & 241 & 245 \\
3 & 2446 & 292 & 301 & 296 \\
4 & 2345 & 363 & 359 & 361 \\
5 & 2247 & 402 & 398 & 400 \\
6 & 2143 & 470 & 482 & 476 \\
7 & 2044 & 502 & 511 & 506 \\
8 & 1945 & 580 & 574 & 577 \\
9 & 1843 & 610 & 603 & 606 \\
10 & 1742 & 741 & 745 & 743 \\
11 & 1645 & 796 & 801 & 798 \\
\hline \hline
\end{tabular}

In the second experiment (i.e. experiment-2), which is described in Fig. 5; tests were also carried out at a lowaltitude flight of about 4.5 meters above a section, as measured by the barometer sensor in the UAV. The length of this section simulates an oil and gas pipeline with no leakage. This simple setup is used to emulate the real-life application of the developed UAV system. Here, the variation of aerial VOC concentration, $C_{g}$ with distance, $L$ (max. $120 \mathrm{~m}$ ) was measured and analyzed as the UAV (with the LDU onboard) traverses along this section in 10 minutes. In the control experiment as described in Fig. 6, three leaking LPG storage cylinders were introduced at three successive positions along the length of the same section (to simulate a leaking pipeline).

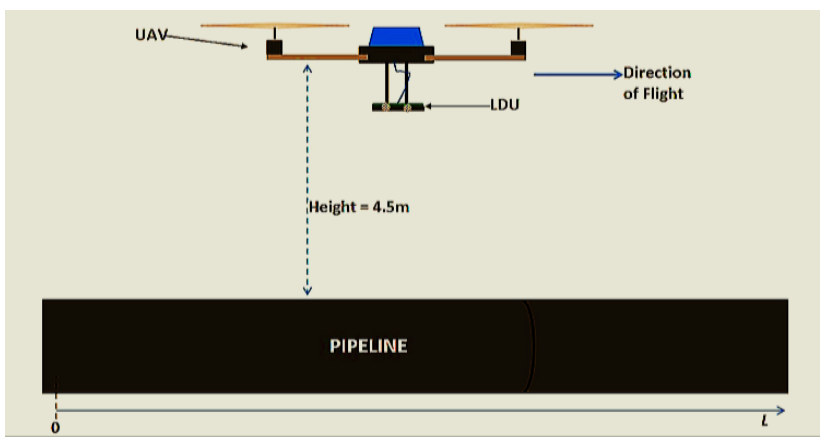

Fig. 5 Setup for experiment-2 (no leakage).

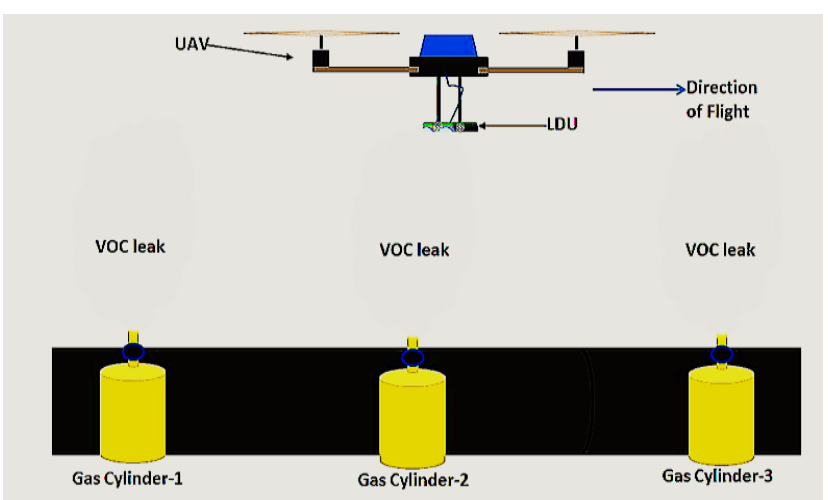

Fig. 6 Control version of experiment-2 (leakage present)

\section{RESULTS AND DISCUSSION}

The results of experiment- 1 as presented in Table1 is plotted in Fig. 7. From this, it is obvious that the concentration of LPG vapor in the air varies inversely as the pressure in the LPG cylinder as expressed. The results of experiment- 2 and its control experiment are plotted in Fig. 8. These reveal that the value of $C_{g}$ is relatively constant throughout the length of the test section in the absence of LPG leakage. The little variation is attributed to ambient influences like smoke, heat and volatile gases from other sources. In the control version of the experiment, a significant increase in the value of $C_{g}$ is detected around the three points along the pipeline route as evident in Fig. 8. These points coincide with the positions where the leaking gas cylinders are stationed. From these analyses, it can be deduced that the developed LDU onboard the UAV is capable of real-time response and high sensitivity to changes in the VOC concentration in a given environment.

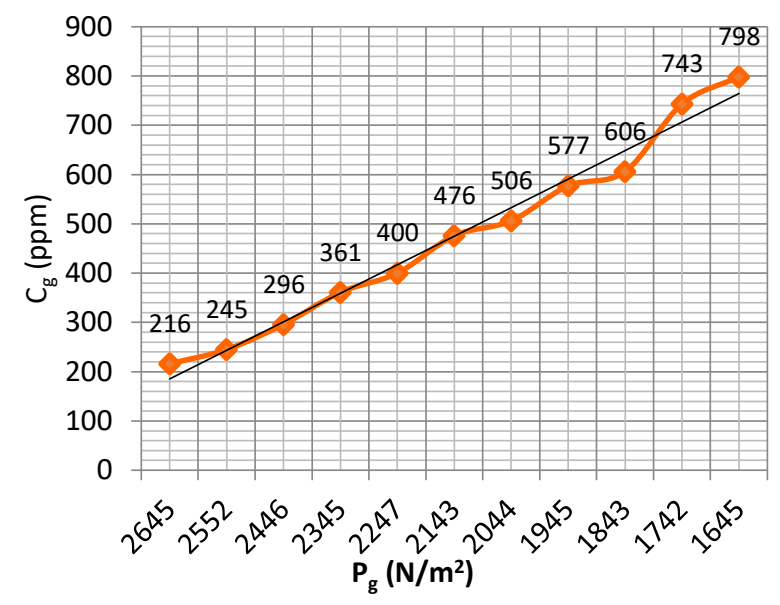

Fig. 7. Plot of $P_{g}$ vs $C_{g}$ in Experiment-1

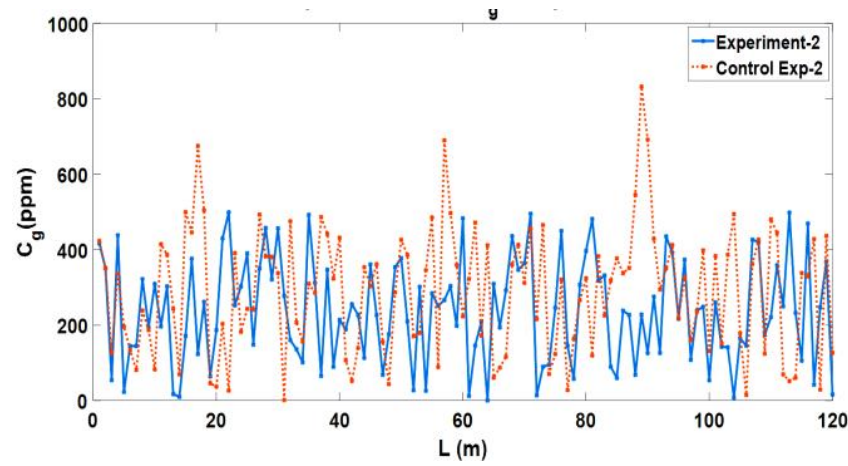

Fig. 8. Plot of L vs. $\mathrm{C}_{\mathrm{g}}$ in Experimet-2 


\section{CONCLUSION}

This research project is implemented using only the available materials. A $22.36 \mathrm{Kg}$ UAV with onboard instrumentation payload is developed for remote monitoring of oil and gas pipeline infrastructures in real-time. In practice, the developed UAV could at full throttle, lift a maximum total mass of $54.42 \mathrm{~kg}$ (its weight plus that of additional payload), compared to the estimated mass limit of $74.922 \mathrm{~kg}$. This UAV system has performed as expected in spite of technical setbacks in the development process. Hence, we are optimistic that our device and measurement technique can be used to facilitate situational awareness and decision making in the oil and gas industry.

\section{REFERENCES}

[1] Omodanisia, E. O., Eludoyin, A. O. and Salamia, A. T., "A multiperspective view of the effects of a pipeline explosion in Nigeria," International Journal of Disaster Risk Reduction, vol. 7, pp. 68-77, 16 May 2014.

[2] Kochetkova, L. I., "Pipeline monitoring with unmanned aerial vehicles," Journal of Physics: Conference Series, 2018.

[3] Austin, R., Unmanned Aircraft Systems: UAV Design, Development and Deployment, West Sussex: John Wiley and Sons Ltd, 2010.

[4] Cristina Gómez and David R. Green, "Small unmanned airborne systems to support oil and gas pipeline monitoring and mapping," Arabian Journal of Geosciences, vol. 10, no. 202, 2017.

[5] Villa, T. F., Gonzalez, F., Miljievic, B., Ristovski, Z. D., and Morawska, L., "An Overview of Small Unmanned Aerial Vehicles for Air Quality Measurements: Present Applications and Future Prospectives," Sensors, vol. 16, no. 7, 2016.

[6] Ejofodomi, O. A. and Ofualagba G., "Exploring the Feasibility of Robotic Pipeline Surveillance for Detecting Crude Oil Spills in the Niger Delta," International Journal of Unmanned Systems Engineering, vol. 5, no. 3, pp. 38-52, 2017.

[7] Ramon, B., Miguel, A., and Juan, M., "Design of an Infrared Imaging System for Robotic Inspection of Gas Leaks in Industria Environments," International Journal of Advanced Robotic Systems, 2015.

[8] Kelvin, N., "Mobile Remote Sensing: A Heterogeneous Linear Sensor Network for Trans-Alaskan Pipeline Leak Detection," Duke University, Durham, North Carolina, 2015.

[9] Omijeh, B. O., Oden, V. M., Joseph, K. J. and Erameh, J. A., "Design and Construction of a Quadcopter with Payload for Pipeline Inspection and Surveillance," Intl. J. of Advanced Research in Electrical, Electronics and Instrumentation Engineering, vol. 5, no. 6, 2016.

[10] Kotarski, D., Benić, Z. and Krznar, M., "Control Design for Unmanned Aerial Vehicles with Four Motors," Interdisciplinary Description of complex System, vol. 14, no. 2, pp. 236-245, 2016.

[11] Mohd, K., "Quadcopter Flight Dynamics," International Journal of Scientific and Technology Research, vol. 3, no. 8, 2014.

[12] Jaaniso, R. and Tan, O. K., Semiconductor Gas Sensors, 2 ed., Woodhead Publishing, 2019 